\title{
Beyond growth signaling: Paneth cells metabolically support ISCs
}

\author{
Cell Research (2017) 27:851-852. doi:10.1038/cr.2017.59; published online 21 April 2017
}

Single Lgr5 $^{+}$intestinal stem cells (ISCs) can be expanded in vitro into epithelial organoids or "mini-guts", self-organizing cellular structures that recreate the intestinal differentiation program; Paneth cells, which constitute the intestinal stem cell niche, secrete stem cell growth signals, and are thus essential for this process. In a recent paper published in Nature, Rodríguez-Colman et al. describe how Paneth cells may be supporting the metabolic state of ISCs.

The small intestinal epithelium is the fastest self-renewing tissue in mammals and has served as the principle model for the study of adult stem cell biology [1]. The small intestine is structurally organized into stem cell compartments, the crypts of Lieberkühn, and functional epithelial compartments, luminal protrusions called villi that extend out from the crypts and contain specialized differentiated epithelial cell types (Figure 1A). Intestinal stem cells (ISCs), which are called crypt base columnar cells (CBCs) and express Lgr5, reside at the base of the crypts intermingled with post-mitotic Paneth cells. The close interaction of CBCs with Paneth cells is essential for maintaining the stem cell function of CBCs. In addition to secreting antibacterial peptides, Paneth cells support stem cell function through the secretion of growth signaling molecules that are required for $\mathrm{CBC}$ proliferation and maintenance [2].

The dependence of CBCs on Paneth cell-mediated paracrine signaling can be recapitulated in vitro. Single Lgr5 ${ }^{+} \mathrm{CBCs}$ can be expanded in vitro in a $3-\mathrm{D}$, matrigel-based culture that results in organoids or "mini-guts", self-organizing cellular structures that recreate the intestinal crypt-villus architecture and differentiation program. While the outgrowth efficiency of single $\mathrm{Lgr}^{+} \mathrm{CBC}$ s is quite low, when plated together with Paneth cells, their outgrowth efficiency increases by 10 -fold [2]. Thus, the organoid system provides a valuable in vitro system for the study of Paneth cell-CBC interactions and interdependencies.

In a paper recently published in $\mathrm{Na}$ ture, Rodríguez-Colman and colleagues used this in vitro intestinal organoid system to elucidate the metabolic interactions between Paneth cells and CBCs [3]. Through metabolite profiling of $\mathrm{Lgr5}^{+} \mathrm{CBCs}$ and Paneth cells isolated from the small intestines of mice, the authors found that these cell populations show distinct metabolic profiles. While the metabolite content of Paneth cells was consistent with a primarily glycolytic metabolic program, that of CBCs was consistent with an oxidative metabolic program. Mitochondria are the cellular site of oxidative metabolism and the authors convincingly show that CBCs have more mitochondria than Paneth cells and are more sensitive to inhibitors of oxidative metabolism (Figure 1B).

The observation that the post-mitotic Paneth cells, but not the highly proliferative $\mathrm{CBCs}$, display features of high glycolytic flux may initially seem counterintuitive. Many proliferative cell types including activated lymphocytes, progenitor cells in the developing retina, cells of the preimplantation stage embryo [4], and tumor cells are highly glycolytic [5]. The correlation between high glycolytic activity and proliferation has been attributed to the increased demand for anabolic metabolism - the biosynthesis of macromolecules which can be supported, in part, by increased levels of glycolytic intermediates that can be used in a number of biosynthetic reactions [5]. In light of this, the primarily glycolytic program of Paneth cells is consistent with the fact that these cells produce and secrete a notable number of signaling molecules [6] and therefore experience a high metabolic demand for the biosynthesis of these molecules.

Likewise, the observation that CBCs have an increased dependence on oxidative metabolism may seem counter to the data from studies that have shown reduced oxidative capacity in embryonic stem cells, long-term hematopoietic stem cells, mesenchymal stem cells, and hepatic stem cells [4]. However, some cancer stem cell populations have been shown to be dependent on oxidative and mitochondrial metabolism. Subpopulations of pancreatic cancer [7], breast cancer [8], melanoma [9], and colorectal cancer [7] have been shown to be preferentially sensitive to drugs such as metformin and oligomycin, which selectively inhibit or perturb oxidative metabolism.

It is possible that dependence on oxidative metabolism buffers CBCs from a variable state of nutrient availability, which can fluctuate between high availability and scarcity. This hypothesis is consistent with data from Rodriguez-Colman et al. showing that lactate excreted from glycolytic Paneth 
A

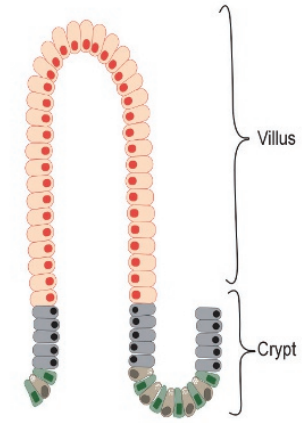

B

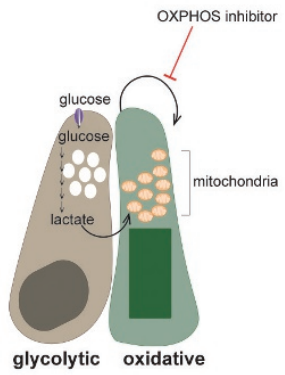

Figure 1 (A) The intestinal epithelium is structurally organized into crypt-villus units. Stem cells and transit-amplifying cells in the crypt proliferate continuously to renew mature cells in the villi. In the crypt, Lgr5 ${ }^{+}$ISCs, CBCs, are intermingled with Paneth cells, differentiated and secretory cells that provide CBCs with growth signals. (B) Paneth cells are highly glycolytic. CBCs have more mitochondria than Paneth cells and have an oxidative metabolic profile. The lactate generated through glycolysis in Paneth cells is excreted and used by CBCs to drive oxidative metabolism, which supports their proliferation and differentiation. $\mathrm{CBCs}$ are dependent on oxidative metabolism as indicated by their sensitivity to inhibitors of oxidative phosphorylation (OXPHOS). In the schematic, orange indicates differentiated cells in villi, grey indicates transit-amplifying progenitor cells, green indicates CBCs, and brown indicates Paneth cells.

cells can be used as a fuel source by the more oxidative CBCs (Figure 1B). This would suggest that Paneth cells are more sensitive to environmental fluctuations in nutrient availability and might therefore provide signaling cues to $\mathrm{CBCs}$ that can induce an adaptation appropriate to the current nutritional state.

This kind of metabolic interdependence is consistent with the previously elucidated response of Paneth cells and $\mathrm{CBCs}$ to caloric restriction [10]. Under conditions of caloric restriction in mice, the intestinal villi shorten and contain fewer differentiated absorptive cells, enterocytes. In contrast, under these conditions, the stem cell compartment expands to contain increased numbers of Paneth cells and CBCs. This Paneth cell-CBC expansion is mediated through decreased activity of the mammalian target of rapamycin complex 1 (mTORC1) in Paneth cells and the consequent Paneth cell release of the paracrine effector, cyclic ADP ribose (cADPR), which promotes $\mathrm{CBC}$ proliferation.

Paradoxically, under conditions of excessive nutrients as is the case for mice fed a long-term high-fat diet
(HFD, a diet consisting of $60 \%$ fat), the number of CBCs also increased but the number of Paneth cells decreased [11]. In this case, CBCs isolated from HFDfed mice were independent of Paneth cells for outgrowth in organoid cultures. The effect of HFD on CBCs was shown to be mediated, in part, by fatty acid-induced activation of a PPAR- $\delta$ transcriptional program, which led to activation of the Wnt/ $/$-catenin pathway in CBCs. While active Wnt signaling in response to HFD can account for the reduced dependency of CBCs on Paneth cells, the question remains as to whether the HFD-induced PPAR- $\delta$ transcriptional program in $\mathrm{CBCs}$ also changes the metabolic profile of these cells. Given the metabolic interdependence of CBCs and Paneth cells described by Rodriguez-Colman et al., it would be informative to investigate how the metabolite profiles of Paneth cells and $\mathrm{CBCs}$ change in response to both caloric restriction and HFD.

Finally, Rodriguez-Colman et al. show that oxidative metabolism in $\mathrm{CBC}$ and the consequent reactive oxygen species (ROS)-induced signaling in these cells drive their differentiation.
The relationship between ROS and stem cell differentiation has been shown in other systems [12], and this provides an example of how metabolism, cell signaling, and gene expression regulation are inextricably linked.

In their study, Rodriguez-Colman et $a l$. show that Paneth cells fuel ISCs not only through growth factor signaling but also by providing them with a metabolic fuel source. By identifying the distinct metabolic programs and therefore potential dependencies of Paneth cells and CBCs, this study highlights how the metabolic demands of any cell are unique to their cellular context and function. In the future, it will be important to understand how the metabolic profiles of these cell types change in response to different organismal stresses and how these responses might be manipulated to promote regeneration, decrease tissue damage, or to prevent tumorigenesis.

\section{Talya L Dayton ${ }^{1}$, Hans Clevers ${ }^{1}$}

${ }^{1}$ Hubrecht Institute, Royal Academy of Arts and Science (KNAW) and University Medical Centre Utrecht, Uppsalalaan 8, 3584CT, Utrecht, The Netherlands

Correspondence: Hans Clevers

E-mail: h.clevers@hubrecht.eu

\section{References}

1 Stange DE, Clevers H. Stem Cells 2013; 31:2287-2295.

2 Sato T, van Es JH, Snippert HJ, et al. Nature 2011; 469:415-418.

3 Rodríguez-Colman MJ, Schewe M, Meerlo M, et al. Nature 2017; 543:424-427.

4 Folmes CD, Dzeja PP, Nelson TJ, et al. Cell Stem Cell 2012; 11:596-606.

5 Lunt SY, Vander Heiden MG. Annu Rev Cell Dev Biol 2012; 27:441-464.

6 Clevers HC, Bevins CL. Annu Rev Physiol 2013; 75:289-311.

7 Sancho P, Barneda D, Heeschen C. $B r J$ Cancer 2016; 114:1305-1312.

8 Hirsch HA, Iliopoulos D, Tsichlis PN, et al. Cancer Res 2009; 69:7507-7511.

9 Roesch A, Vultur A, Bogeski I, et al. Cancer Cell 2013; 23:811-825.

10 Yilmaz ÖH, Katajisto P, Lamming DW, et al. Nature 2012; 486:490-495.

11 Beyaz S, Mana MD, Roper J, et al. Nature 2016; 531:53-58.

12 Chandel NS, Jasper H, Ho TT, et al. Nat Cell Biol 2016; 18:823-832. 